\title{
Drag-reduction of 3D printed shark-skin-like surfaces
}

\author{
Wei DAI ${ }^{1}$, Masfer ALKAHTANI ${ }^{2,3}$, Philip R. HEMMER ${ }^{2,4}$, Hong LIANG ${ }^{1, *}$ \\ ${ }^{I}$ Department of Mechanical Engineering, Texas A\&M University, College Station, TX 77843, USA \\ ${ }^{2}$ Institute for Quantum Science and Engineering (IQSE), Texas A\&M University, College Station, TX 77843, USA \\ ${ }^{3}$ Center for Quantum Optics and Quantum Informatics, KACST, Riyadh 11442, Saudi Arabia \\ ${ }^{4}$ Department of Electrical and Computer Engineering, Texas A\&M University, College Station, TX 77843, USA \\ Received: 18 October 2017 / Revised: 05 February 2018 / Accepted: 07 September 2018 \\ (C) The author(s) 2018. This article is published with open access at Springerlink.com
}

\begin{abstract}
The marvels of the slippery and clean sharkskin have inspired the development of many clinical and engineering products, although the mechanisms of interfacial interaction between the sharkskin and water have yet to be fully understood. In the present research, a methodology was developed to evaluate morphological parameters and to enable studying the effects of scale orientation on the fluidic behavior of water. The scale orientation of a shark skin was defined as the angle between the ridges and fluid flow direction. Textured surfaces with a series orientation of scales were designed and fabricated using 3D printing of acrylonitrile butadiene styrene (ABS). The fluid drag performance was evaluated using a rheometer. Results showed that the shark-skin-like surface with 90 degree orientation of scales exhibited the lowest viscosity drag. Its maximum viscosity reduction was $9 \%$. A viscosity map was constructed based on the principals of fluid dynamic. It revealed that the drag reduction effect of a shark-skin-like surface was attributed to the low velocity gradient. This was further proven using diamond nitrogen-vacancy sensing where florescent diamond particles were distributed evenly when the velocity gradient was at the lowest. This understanding could be used as guidance for future surface design.
\end{abstract}

Keywords: shark skin; 3D printing; textured surface; drag reduction; nitrogen vacancy

\section{Introduction}

The natural wonders of the sharkskin have attracted great attention in past decades. The slippery and textured shark skin has inspired biologists and engineers to design product with like surfaces for better performance. Examples have been found in products ranging from swim suits to air planes. The hydrodynamic behavior of a surface with shark-skin morphology has been reported to be improved [1]. A shark-skin-like surface was composed of textured shark scales with many micrometer sized grooves and ridges. In different parts of a shark's body, like head, dorsal or anal fin, their scales dimensions vary. Despite the wide diversity of shark skins, a large amount of work has been reported trying to develop a simplified model. Such models have considered cross sectional profiles including blade [2-4], saw tooth [5-7], and scallop [8-10]. The ridge geometry could be optimized for better performance by alternating height, spacing, thickness, and yaw angle. In prior work optimizing morphological parameters in drag reduction [11-13], the best combination was the blade shape of 0.5 in height-to-spacing ratio. Under this condition the maximum drag reduction of $9.9 \%$ was obtained [14]. This work is based on the design of blade-shaped shark-skin-like surfaces.

The drag-reduction mechanism of shark-skin-like surfaces has been studied extensively in recent years. Theoretical analysis has been focused on the velocity profile and pressure drop in the fluid flow over the surface through numerical simulation [15-17].

* Corresponding author: Hong LIANG, E-mail: hliang@tamu.edu 
Experimental methods have been reported using a wind tunnel and a fluid flow channel [18-20]. Such methods are unique but rather costly. In the present research, a new method to evaluate the performance of textured surface was proposed. Performance of water against shark-skinned surfaces was evaluated using a rheometer. The shear stress and viscosity of fluid flows over the surface were measured in situ. In an effort to explain the mechanisms of lubrication on a textured surface, we used diamond nanoparticles as a marker in the flowing fluid. Nanoparticles have been widely applied as additives [21-24] and tracking particles $[25,26]$ in the lubricating study. The distribution of nanoparticles is the indication of the overall and instantaneous fluid filed of scalars and vectors, reflecting the behavior of fluid [27]. The distribution of diamond particles on the shark-skinned surface were characterized thorough nitrogen-vacancy centers sensing [28]. Diamond particles are exceptionally robust dyes used in bio imaging $[29,30]$. Here the distribution of diamond particle would facilitate our investigation in velocity gradient analysis and the drag reduction mechanism of shark-skin surfaces.

Using 3D printing method to generate surfaces with shark-skin textures has many advantages. Conventional milling and molding are routinely used to fabricate shark-skinned surfaces that need multistep [31]. In addition to complexity, they are expensive and lack dimensional control. In comparison, 3D printing is a facile and cost effective method that can fabricate shark skin scales [12]. In this research, three shark-skin-like surfaces with different scale orientation were designed and $3 \mathrm{D}$ printed. Despite numerous studies on the optimization of morphology and dimension, few reports considered the effect of shark skin scale orientation on fluid flow. Since the flow condition is complicated and unpredicted in real world, it is necessary to optimize the orientation of shark skin scale to realize significant drag reduction in hydrodynamic lubrication.

\section{Materials and methods}

Design and dimensions of a single shark skin scale are illustrated in Fig. 1. The height of ridges was $400 \mu \mathrm{m}$. The diameter of the textured surface was $25 \mathrm{~mm}$. Scale orientation is defined as the angle between the ridges and fluid flow direction, which is circumferential. To evaluate the sharkskin performance with a different scale orientation, three textured surface were designed using Solid Works (Solid Works Corp., Waltham, MA, USA) as shown in Fig. 1. The smooth surface was measured as well for comparison.

Shark-skin-like surfaces were fabricated using a Flash Forge Dreamer dual extrusion 3D printer. To mimic the surface feature of a shark's skin, we used the smallest nozzle that produced smallest width of the ridge of $400 \mu \mathrm{m}, 2-3$ times bigger than the average length of a real scale. The layer thickness ranges from $100 \mu \mathrm{m}$ to $400 \mu \mathrm{m}$. The roughness of printed smooth surface is $30 \mu \mathrm{m}$. The polymer for $3 \mathrm{D}$ printing is
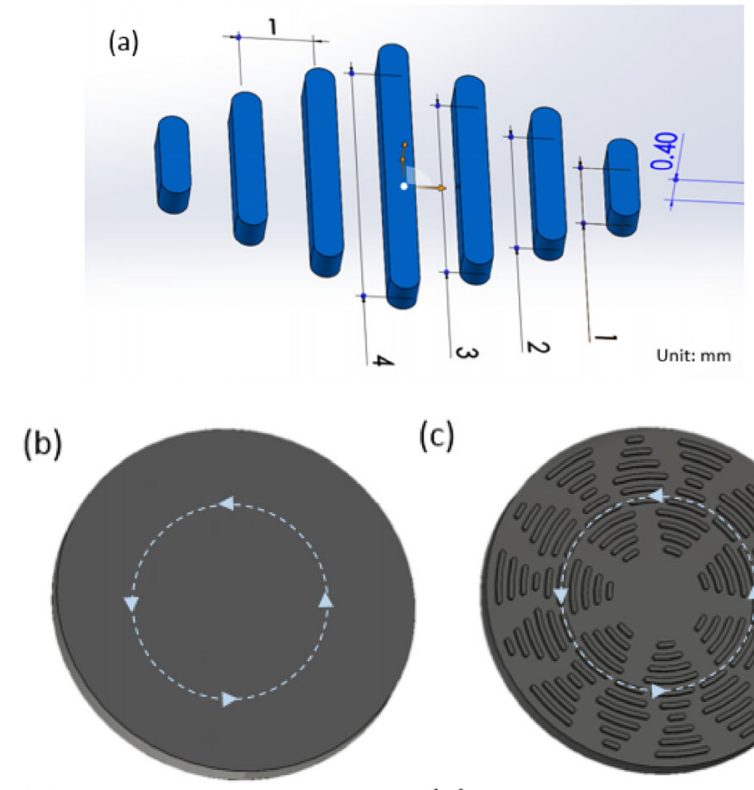

(c) (d)

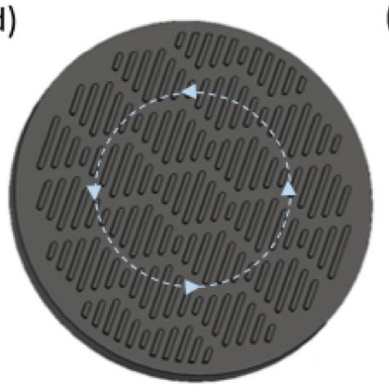

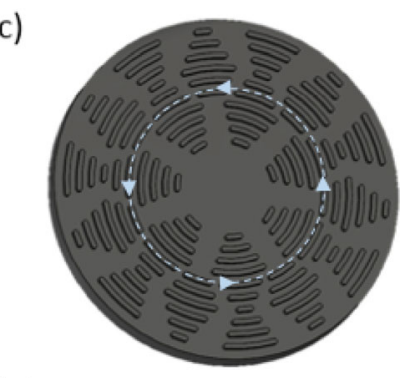

(e)

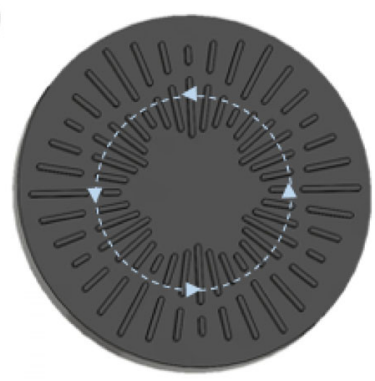

Fig. 1 Design and dimensions of shark-skin-like surface: single scale of sharkskin (the direction of water flow as indicated by the dash line): (a) a simplified model of shark-skinned surface; (b) a smooth surface for comparison; (c) a textured surface with parallel shark-skin scale orientation (0 degree); (d) a textured surface with shark skin mix scale orientation (45 degree); (e) a textured surfaces with perpendicular shark-skin scale orientation (90 degree). 
acrylonitrile butadiene styrene (ABS). Melting temperature is $105^{\circ} \mathrm{C}$ and tensile strength is $34 \mathrm{GPa}-38 \mathrm{GPa}$. Rigid surface will maintain the shape during the fluidic test. Printing temperature is around $220^{\circ} \mathrm{C}$.

Effects of drag reduction were studied through measuring the viscosity of fluid flowing over various surfaces. The viscosity of deionized (DI) water containing $0.1 \mathrm{wt} \%$ diamond particles was measured using an AR-G2 rheometer (TA instruments) under the ambient temperature $\left(25^{\circ} \mathrm{C}\right)$. The lowest torque can be measured is $0.01 \mu \mathrm{N} \cdot \mathrm{m}$. The schematic illustration of rheometer was shown in Fig. 2. We found some water would splash out of the textured surface under a high shear rate in the old design (Fig. 2(a)). In this case, the liquid volume would decrease and an air gap would exist between the upper spindle and the textured surface, which leads to a high error to the shear stress and viscosity. A new design for textured surface viscosity measurement was shown in Fig. 2(b). The extra container would make sure there was a constant liquid volume during the viscosity measurement. Each test $10 \mathrm{ml}$ water was added in the container to eliminate the air gap. Under a high shear rate $\left(>400 \mathrm{~s}^{-1}\right)$, the liquid level will become a cone shape due to the centrifugal force as shown in Fig. 2(b). DI water will avoid the interference of impurity during viscosity measurement. Diamond particles are florescent and were used as markers representing the fluidic performance. Particles were uniformly distributed in water. Textured surfaces were fixed on the lower plate. The gap between the spindle $(\Phi=25 \mathrm{~mm})$ and the textured surface was set at $3 \mathrm{~mm}$ and was fully filled with water. The shear rate ranged from $0 \sim 700 \mathrm{~s}^{-1}$ to cover the regime from laminar flow to turbulent flow. The shear stress and other parameters were recorded simultaneously. The viscosity of the fluid
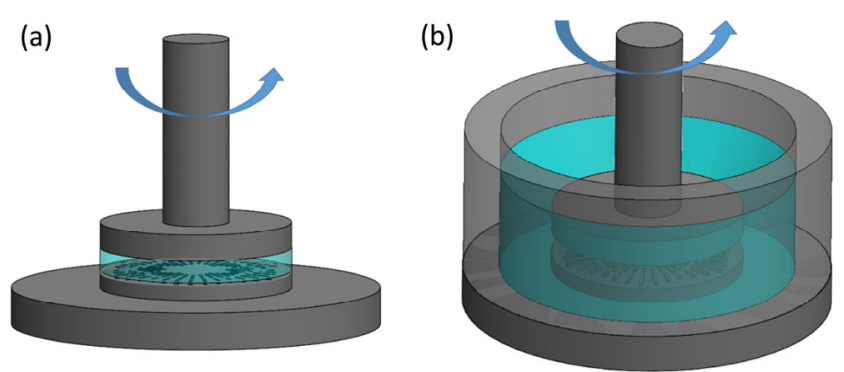

Fig. 2 The schematic illustration of textured surface viscosity measurement. (a) an original design; (b) a new design. was calculated as the ratio of shear stress to shear rate. Each experiment was repeated for at least three times.

The distribution of diamond particles was studied in order to reveal the drag reduction mechanism of shark-skin-like surfaces. After the viscosity measurement, the distribution of diamond particles on shark-skinned surfaces was characterized by using a special home-made big objects laser scanning microscope. This system is designed to scan over a large area $(5 \mathrm{~cm} \times 5 \mathrm{~cm})$ without any restrictions of microscope objectives as shown in Fig. 3. Shark-skinned surfaces were placed at a focal length distance of a big field of view lens and excited with a $532 \mathrm{~nm}$ laser excitation with low power $10 \mathrm{~mW}$. The images of diamonds distributed over the disks were collected and analysed with a homemade spectrometer equipped with a starlight camera (Trius camera model SX-674) and a photon counter (Hamamatsu photon counter model number H7155-21).

\section{Results and discussion}

\subsection{Morphological characteristics}

First, morphological characteristics of shark-skinned surfaces were analyzed based on the ridges spacing and height. Three-dimensionally (3D) printed sharkskinned surfaces were shown in Fig. 4. Designed features were clearly printed. Other than the smooth surface, 3 shark-skinned surfaces with different shark skin scale orientation were designed considering the flowing direction of rheometer spindle. There were several reasons to choose the scale distribution in this study. First, we want to print the shark skin scales on

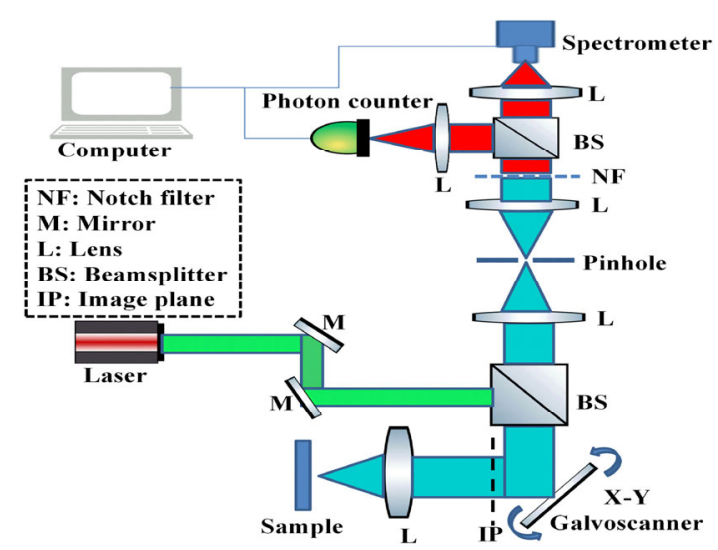

Fig. 3 The setup of a homemade laser scanning microscope. 
(a)

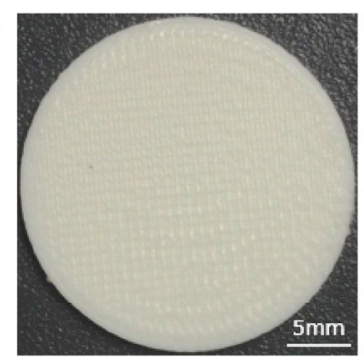

(c)

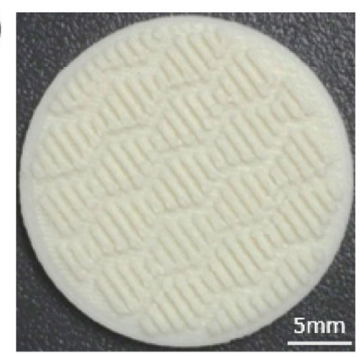

(b)

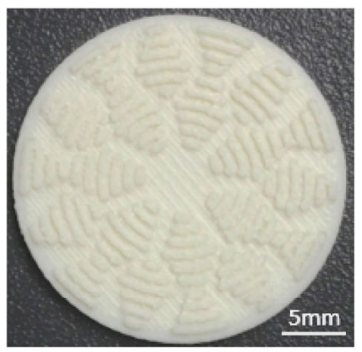

(d)

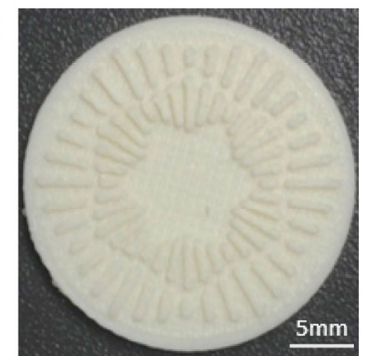

Fig. 4 3D printed shark-skinned surfaces with different scale orientation: (a) smooth; (b) parallel (0 degree); (c) mix (45 degree); and (d) perpendicular (90 degree).

the surface as clearly as possible. In the case of 0 degree and 90 degree, if we print the scale in the center, ridges will interfere with each other and the texture is not clear. So the center regions are smooth in Figs. 1(c) and 1(e). Since we used the rheometer to measure the fluid viscosity on the surface, velocity near the center of the surface is the lowest while periphery is the highest. The measured shear stress is more affected by the texture at the periphery. Therefore the spatial non-uniformity will not affect the viscosity measurement too much. In Fig. 1(e), we want to keep the uniform orientation (90 degree) between the ridges and fluid flow direction. Since the fluid direction is rotational, the ridge direction should be radial. So periphery has a higher ridge spacing than towards near the center. The performance of the shark-skinned surface was closely related with its ridge height (h) and spacing (s). Ridge height is uniform for all the shark-skinned surface, which is $0.4 \mathrm{~mm}$. Ridge space varies for the shark-skinned surface with a different shark skin scale orientation. For the parallel and mix ones, ridge space is $1 \mathrm{~mm}$. For the perpendicular one, ridge space is not uniform, ranging from $1 \sim 2 \mathrm{~mm}$. The average space is $1.5 \mathrm{~mm}$. Therefore, the average height versus spacing ratio $(\mathrm{h} / \mathrm{s})$ for parallel and mix surface is 0.4 . The average $\mathrm{h} / \mathrm{s}$ ratio for the perpendicular surface is $0.4 / 1.5=0.27$.

\subsection{Fluidic performance}

In the next step we investigate the fluidic performance of shark-skinned surfaces to reveal the effect of scale orientation. Figure 5 shows the viscosity of water flowing over different shark-skinned surfaces under different shear rates. The error bars come from the standard deviation of three repeatable experiments. Generally speaking, water is Newtonian fluid, which indicates that its viscosity is constant under various shear rates. Due to the new setup of the rheometer (Fig. 5), however, centrifugal force will increase with the increase of shear rate, thereby leading to higher shear stress. Therefore, the viscosity of water will increase with the increase of shear rate. The single viscosity value has little meaning in this research, but the viscosity reduction effect between the smooth surface and sharkskin like surface is interesting. In Fig. 5, when the shear rate was lower than $300 \mathrm{~s}^{-1}$, the smooth surface showed lower viscosity than sharkskinned surfaces. This indicated laminar flow regime. The surface with the parallel shark skin scale showed the highest viscosity. When the shear rate was higher than $300 \mathrm{~s}^{-1}$, the smooth surface showed a much higher viscosity than shark-skinned surfaces. This indicated the regime of turbulent flow. The surface with the perpendicular shark skin scale showed the lowest viscosity. In the laminar flow regime, ridges of the shark-skinned surface block the movement of fluid, which leads to higher drag. The drag reduction effect

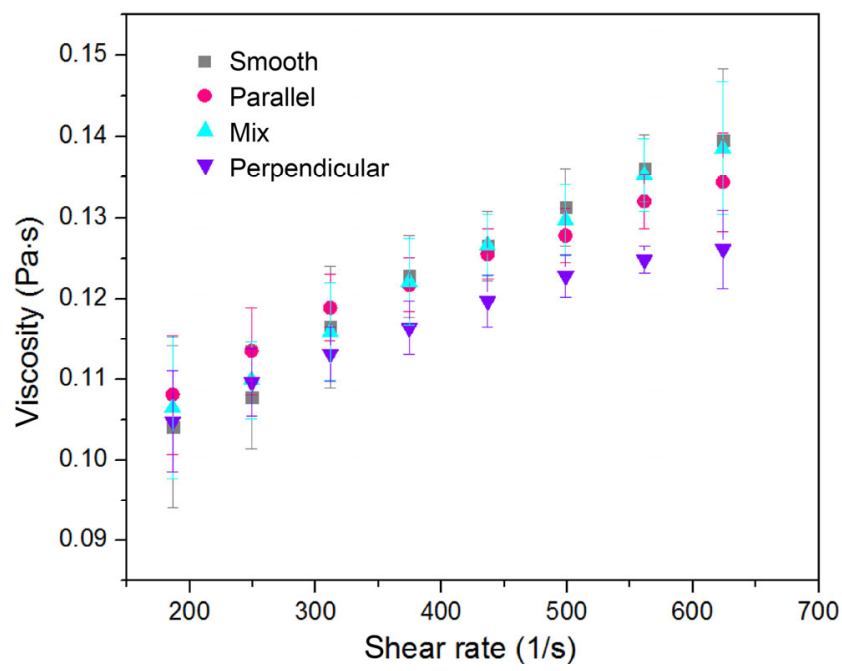

Fig. 5 Viscosity of water flowing over different shark-skinned surfaces under different shear rate. 
of the shark-skinned surface could only be found in the turbulent flow regime. In this regime, fluid drag increased dramatically due to the existence of the vortices. Formation of vortices is complicated and not fully understood. The higher the contact area between the vortices and surface, the higher the shear stress and fluid drag. In the case of a shark-skinned surface, vortices usually exist on the top of the ridges rather than the valleys of ridges. Therefore, vortices only contact the edge of ridges instead of the whole surface, leading to less fluid drag compared with the smooth surface. This mechanism has already been proved by fluid flow visualization [32].

\subsection{The mechanism of drag reduction}

To reveal the drag reduction mechanism of sharkskinned surfaces, the distribution of diamond particles was studied using a laser scanning microscope. After the viscosity test under the shear rate of $400 \mathrm{~s}^{-1}$, the distribution of diamond particles on shark-skinned surfaces with different shark skin orientation were provided in Fig. 6. The 3D particles' distributions were found in Fig. 7 using Image J software. Peak intensity corresponds to the amount of diamond particles on the surface. From the four images, we found the majority of diamond particles were located at the center

(a)

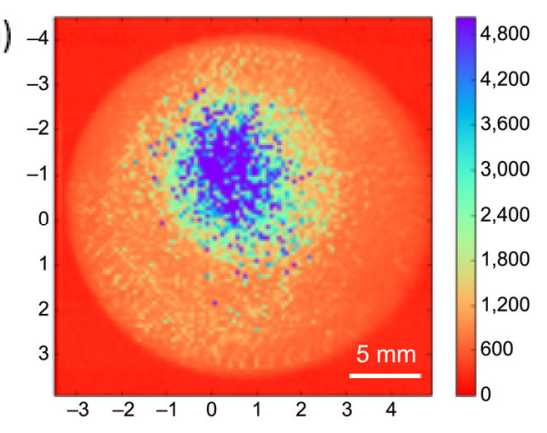

(c)

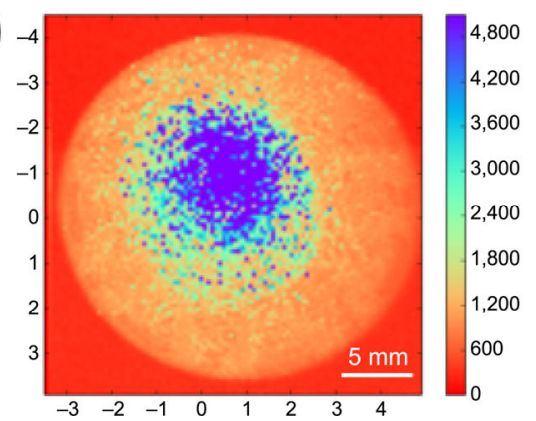

of the surface. This is due to the rotational movement and centrifugal force of the spindle. Spindle center shows the lowest velocity while spindle edge shows the highest velocity. In this case, more diamond particles (higher peak intensity) indicated lower fluid velocity while fewer diamond particles (lower peak intensity) indicated higher fluid velocity. As a result, the distribution of diamond particles could be correlated with the parallel fluid velocity gradient on the shark-skinned surface. Considering the moving space of particles, the normal scale is $3 \mathrm{~mm}$ (the gap between the spindle and surface) and the parallel scale is $25 \mathrm{~mm}$ (surface diameter). The distribution of diamond particles in the parallel scale played a more important role on the drag force. So only the parallel velocity gradient was studied here. Perpendicular scale orientation showed the highest particle distribution uniformity which indicated the lowest velocity gradient.

In order to quantitatively analyze the uniformity of particle distribution, line scan was conducted across the center of the shark skin surfaces. Profiles could be found in Fig. 8. The uniformity of particle distribution was calculated as the reciprocal of deviation of light intensity. Lower deviation of light intensity indicated higher uniformity of particle distribution and lower
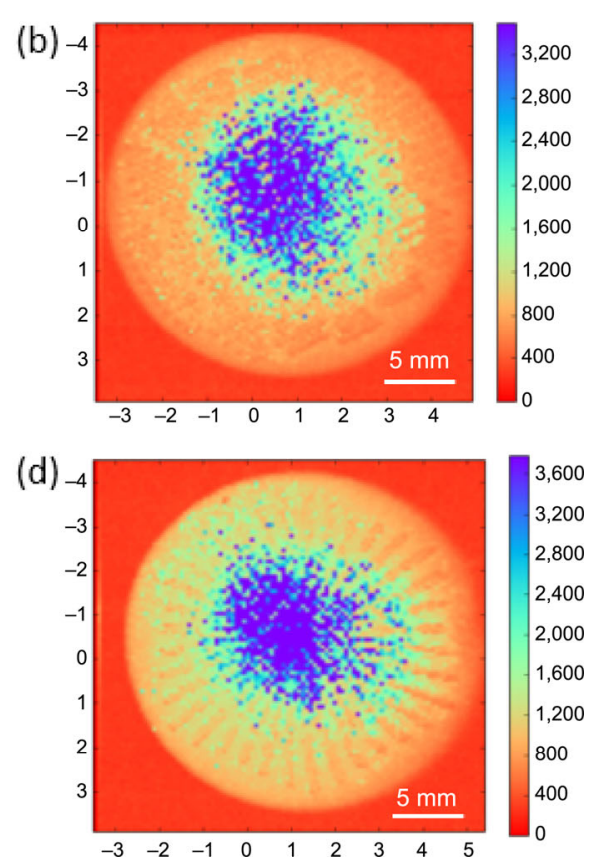

Fig. 6 The distribution of diamond particles on shark-skinned surface with different scale orientation after the viscosity test under $400 \mathrm{~s}^{-1}$ : (a) smooth; (b) parallel (0 degree); (c) mix (45 degree); and (d) perpendicular (90 degree). 


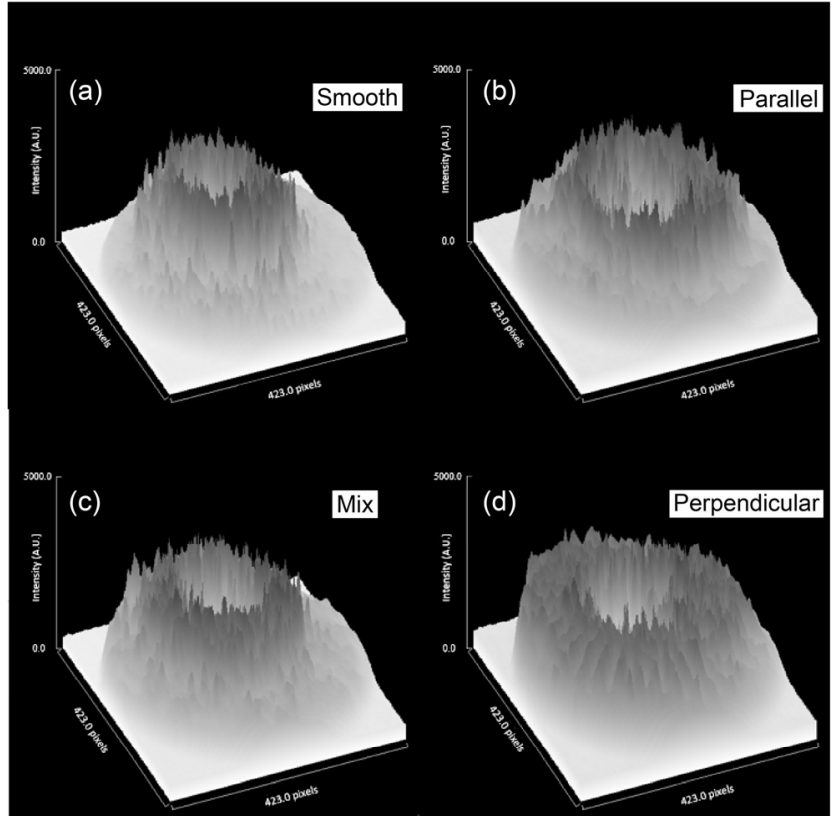

Fig. 7 3D diamond particles distribution on textured surface with different shark skin orientation after the viscosity test under $400 \mathrm{~s}^{-1}$ : (a) smooth; (b) parallel (0 degree); (c) mix (45 degree); and (d) perpendicular (90 degree).

gradient of velocity. At least 4 different line scan of each sample were collected and results could be found in Fig. 9. Perpendicular scale orientation showed the highest uniformity of particle distribution, which indicated the lowest gradient of velocity. The drag is, in fact, a measure of the energy required to transfer momentum between the fluid and the surface. Velocity gradient will create in the adjacent layers of fluid. The lower gradient of velocity will indicated less energy required for momentum transfer between adjacent layers of fluid, which leads to lower drag and viscosity of fluid. The distribution of diamond particle could explain the good reduction effect of viscosity of shark-skinned surfaces experimentally. It is a powerful tool to study the performance of other shark-skinned surfaces as well. The drag is, in fact, a measure of the energy required to transfer momentum between the fluid and the surface. The velocity gradient will create in the adjacent fluid layers. Lower velocity gradient will indicated less energy required for momentum transfer between adjacent fluid layers which leads to lower fluid drag and viscosity. The distribution of diamond particles could explain the good viscosity reduction effect of shark-skinned surfaces experimentally. It is a powerful tool to study the performance of other shark-skinned surfaces as well.

To study the fluidic performance on the smooth surface, Reynolds number is examined against surface parameters. To differentiate the laminar flow regime
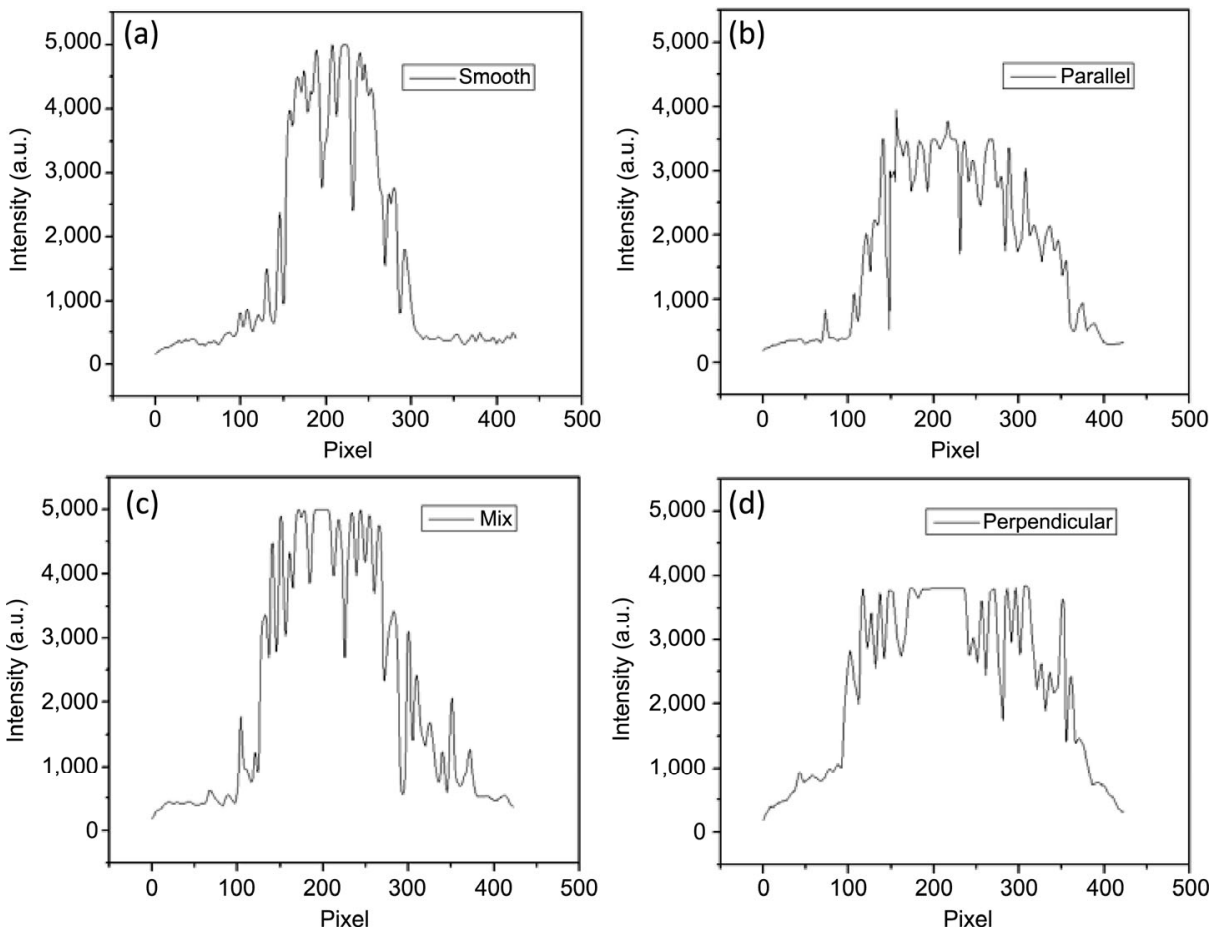

Fig. 8 Line scan profile of diamond particle distribution on surfaces with different scale orientation after the viscosity test under $400 \mathrm{~s}^{-1}$. 


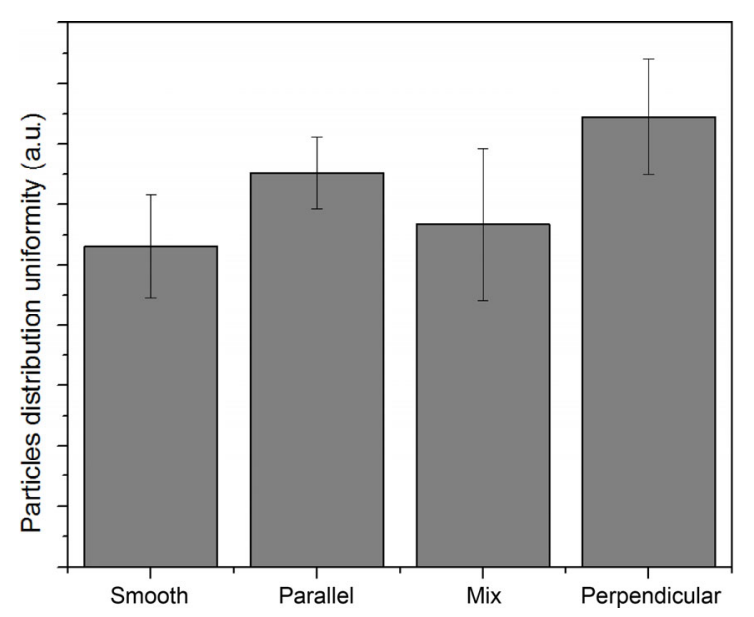

Fig. 9 Diamond particle distribution uniformity comparison of textured surface with different shark skin orientation after the viscosity test under $400 \mathrm{~s}^{-1}$ : (a) smooth; (b) parallel (0 degree); (c) mix (45 degree); and (d) perpendicular (90 degree).

and turbulent flow regime:

$$
R e=\frac{u L}{v}
$$

where $u, L$, and $v$ are fluid velocity, characteristic linear dimension, and fluid kinematic viscosity, respectively. For the fluid flow over a flat plate, the transition Reynolds number is around 500,000. For fluid flow with a lower Reynolds number, the flow is in the laminar regime. For that with the higher Reynolds number, it is in the turbulent regime [33]. Likewise, in order to evaluate the drag reduction performance of shark-skinned surface with different dimensions, the dimensionless parameter $\mathrm{S}^{+}$was introduced. It represents an effective Reynolds number considering ridges' spacing [13]:

$$
S^{+}=\frac{S}{v} \sqrt{\frac{\tau_{\mathrm{w}}}{\rho}}
$$

where $s, \rho, v$ and $\tau_{\mathrm{w}}$ are the spacing between adjacent ridges, fluid density, fluid kinematic viscosity, and average shear stress of the whole shark-skinned surface.

Eventually, in order to analyze the viscosity reduction effect quantitatively, performance of shark-skinned surfaces compared with the smooth surface under various $\mathrm{S}^{+}$number was provided in Fig. 10. $\mathrm{X}$ axis showed the $\mathrm{S}^{+}$number ranging from 0.3 to 0.9 , and $\mathrm{Y}$ axis showed the ratio of shark-skinned surface viscosity to smooth surface viscosity. A ratio is higher or lower than 1, indicating the viscosity increase or decrease

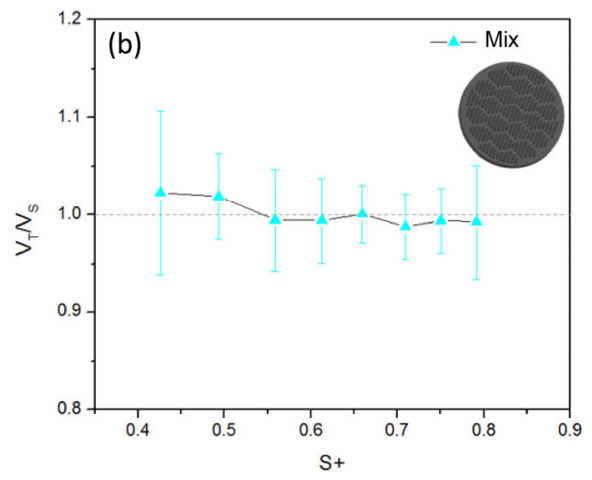

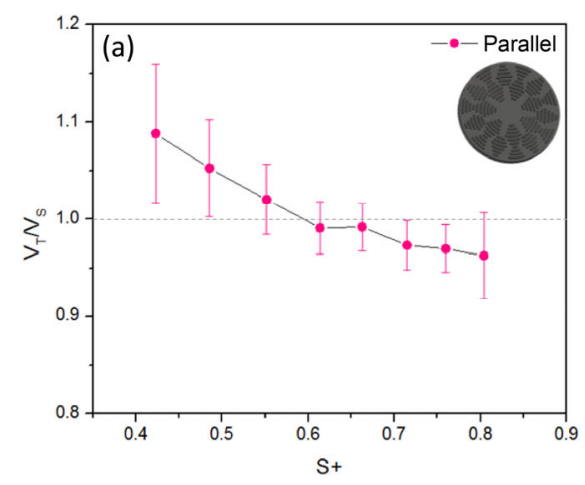

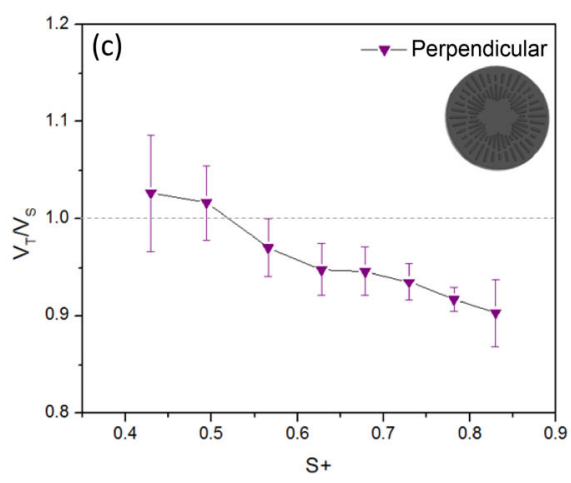

Fig. 10 The viscosity reduction effect of shark-skinned surface compared with smooth surface under various $\mathrm{S}^{+}$number: (a) parallel; (b) mix; and (c) perpendicular. 
effect of shark-skinned surface respectively. All the three shark-skinned surfaces showed a similar trend. Viscosity reduction effect was getting more and more significant with the increase of $\mathrm{S}^{+}$number. In the case of the parallel oriented shark-skinned surface, the $S^{+}$ transition number was 0.6. The mix oriented sharkskinned surface didn't show too much viscosity reduction effect, the $\mathrm{S}^{+}$transition number was around 0.55 . For the case of the perpendicular oriented shark-skinned surface, the $\mathrm{S}^{+}$transition number was 0.52 . The error is $4.5 \%$. Considering the orientation of shark skin ridges upon the fluid flow direction, we can label the parallel, mix, and perpendicular pattern as 0 degree, 45 degree, and 90 degree, respectively. Eventually, the viscosity map of the shark-skinned surface considering the orientation and $\mathrm{S}^{+}$number could be concluded in Fig. 11. In the red regime, viscosity of the shark-skinned surface would increase compared with the smooth surface while it would decrease in the blue regime. The shark-skinned surface with 90 degree orientation showed the largest viscosity reduction. The maximum viscosity reduction is $9 \%$. In the literature, under the high $\mathrm{S}^{+}$number (5 35), the drag reduction decreases as the angle between the pattern and flow direction increases [11]. It doesn't conflict with our results, since the shark-skinned surface was evaluated under small $\mathrm{S}^{+}$number $(0.3 \sim 1)$ in this research. Since not much data was reported in this range, we choose $\mathrm{S}^{+}$from 0.3 to 1 . In this viscosity map, we could estimate the behavior of the shark-skinned surface with different dimensions, scale orientation, and flow conditions. Moreover, a design of the shark-

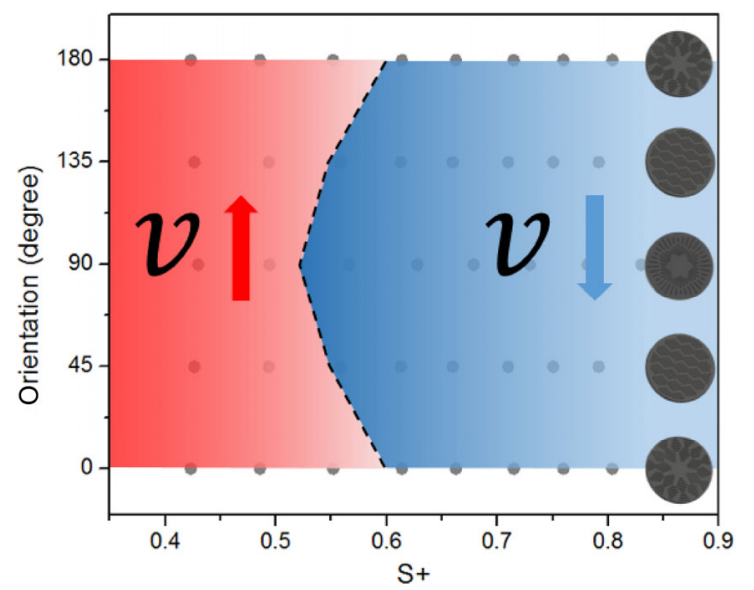

Fig. 11 The viscosity map of shark-skinned surface with different shark skin orientation (from 0 degree to 90 degree). skinned surface with better performance could be achieved using this map.

\section{Conclusions}

In this research, effects of scale orientation on the performance of shark-skin-like surfaces were studied. Compared with the smooth surface, the shark-skinned surface with 90 degree orientation of scales showed the lowest viscosity drag. The maximum viscosity reduction was $9 \%$. This was further proven by the diamond nitrogen-vacancy sensing experiments. Diamond particles worked as tracking particles in fluid. Their distributions were found closely related with the surface velocity gradients. The shark-skinned surface with a 90 degree orientation scale showed a more uniform particles' distribution which indicated a lower velocity gradient. Less momentum transfer between adjacent fluid layers leads to a lower fluid drag. Eventually, a viscosity map of the shark-skinned surface with a different scale orientation was created. This will facilitate the design of a shark-skinned surface with better performance. The understanding generated in this study could be used as a guideline for future study in surface design and texturing.

\section{Acknowledgement}

Part of this research was sponsored by the Turbomachinery Laboratory, Texas A\&M Engineering.

Open Access: The articles published in this journal are distributed under the terms of the Creative Commons Attribution 4.0 International License (http:// creativecommons.org/licenses/by/4.0/), which permits unrestricted use, distribution, and reproduction in any medium, provided you give appropriate credit to the original author(s) and the source, provide a link to the Creative Commons license, and indicate if changes were made.

\section{References}

[1] Luo Y H, Zhang D Y, Liu Y F, Li Y Y, Ng E Y K. Chemical, mechanical and hydrodynamic properties research on composite drag reduction surface based on biological 
sharkskin morphology and mucus nanolong chain. $J$ Mechan Med Biol 15(5): 1550084 (2015)

[2] Bechert D W, Bartenwerfer M. The viscous flow on surfaces with longitudinal ribs. J Fluid Mechan 206: 105-129 (1989)

[3] Büttner C C, Schulz U. Shark skin inspired riblet structures as aerodynamically optimized high temperaturecoatings for blades of aeroengines. Smart Mater Struct 20(9): 094016 (2011)

[4] Lang A, Habegger M L, Motta P. Shark skin drag reduction. In Encyclopedia of Nanotechnology. Bhushan B, Ed. Dordrecht: Springer, 2012: 2394-2400.

[5] Bechert D W, Bartenwerfer M, Hoppe G, Reif W-E. Drag reduction mechanisms derived from shark skin. In Proceedings of the 15th ICASCongress, London, England, 1986: 1044-1068.

[6] Chen H W, Zhang X, Ma L X, Che D, Zhang D Y, Sudarshan $\mathrm{T}$ S. Investigation on large-area fabrication of vivid shark skin with superior surface functions. Appl Surface Sci 316: 124-131 (2014)

[7] Sudo S, Tsuyuki K, Ito Y, Ikohagi T. A study on the surface shape of fish scales. JSME Int J Ser C 45(4): 1100-1105 (2002)

[8] Anderson E J, MacGillivray P S, DeMont M E. Scallop shells exhibit optimization of riblet dimensions for drag reduction. Biol Bull 192(3): 341-344 (1997)

[9] El-Samni O A, Chun H H, Yoon H S. Drag reduction of turbulent flow over thin rectangular riblets. Int J Eng Sci 45(2-8): 436-454 (2007)

[10] Abdulbari H A, Yunus R M, Abdurahman N H, Charles A. Going against the flow-A review of non-additive means of drag reduction. J Ind Eng Chem 19(1): 27-36 (2013)

[11] Dean B, Bhushan B. Shark-skin surfaces for fluid-drag reduction in turbulent flow: A review. Philos Trans Roy Soc London A: Math Phys Eng Sci 368(1929): 4775-4806 (2010)

[12] Wen L, Weaver J C, Lauder G V. Biomimetic shark skin: Design, fabrication and hydrodynamic function. $J$ Exp Biol 217(10): 1656-1666 (2014)

[13] Walsh M J. Riblets as a viscous drag reduction technique. AI A A J 21(4): 485-486 (1983)

[14] Bechert D, Bruse M, Hage W, Meyer R, Bechert D, Bruse M, Hage W, Meyer R. Biological surfaces and their technological application-laboratory and flight experiments on drag reduction and separation control. In Proceedings of the 28th Fluid Dynamicsand Co-located Conference, Snowmass Village, CO, USA, 1997: 1960.

[15] Goldstein D, Handler R, Sirovich L. Direct numerical simulation of turbulent flow over a modelled riblet-covered surface. J Fluid Mechan 302: 333-376 (1995)

[16] Zhang D-Y, Luo Y-H, Li X, Chen H-W. Numerical simulation and experimental study of drag-reducing surface of a real shark skin. J Hydrodyn Ser B 23(2): 204-211 (2011)
[17] Bixler G D, Bhushan B. Bioinspired rice leaf and butterfly wing surface structures combining shark skin and lotus effects. Soft Matter 8(44): 11271-11284 (2012)

[18] Venet C, Vergnes B. Experimental characterization of sharkskin in polyethylenes. J Rheol 41(4): 873-892 (1997)

[19] Popovich A T, Hummel R L. Experimental study of the viscous sublayer in turbulent pipe flow. AI Ch $E J$ 13(5): 854-860 (1967)

[20] Wang J-J, Lan S-L,Chen G. Experimental study on the turbulent boundary layer flow over riblets surface. Fluid Dyn Res 27(4): 217-229 (2000)

[21] Dai W, Lee K, Sinyukov A M, Liang H. Effects of vanadium oxide nanoparticles on friction and wear reduction. $J$ Tribol 139(6): 061607 (2017)

[22] Dai W, Kheireddin B, Gao H, Liang H. Roles of nanoparticles in oil lubrication. Tribol Int 102: 88-98 (2016)

[23] Dai W, Kheireddin B, Gao H, Kan Y W, Clearfield A, Liang H. Formation of anti-wear tribofilms via $\alpha-\mathrm{ZrP}$ nanoplatelet as lubricant additives. Lubricants 4(3): 28 (2016)

[24] Xiao H P, Dai W, Kan Y W, Clearfield A, Liang H. Amine-intercalated $\alpha$-zirconium phosphates as lubricant additives. Appl Surface Sci 329: 384-389 (2015)

[25] Dai W, Chen Y Y, Lee K, Sinyukov A M, Alkahtani M, Hemmer P R, Liang H. In situ investigation of the growth of a tribofilm consisting of $\mathrm{NaYF}_{4}$ fluorescent nanoparticles. Tribol Trans 61(3): 503-512 (2018)

[26] Sanchez C, Chen Y Y, Parkinson D Y, Liang H. In situ probing of stress-induced nanoparticle dispersion and friction reduction in lubricating grease. Tribol Int 111: 66-72 (2017).

[27] Tropea C, Yarin A, Foss J F. Springer Handbook of Experimental Fluid Mechanics. Berlin Heidelberg: Springer Science \& Business Media, 2007.

[28] McGuinness L P, Yan Y, Stacey A, Simpson D A, Hall L T, Maclaurin D, Prawer S, Mulvaney P, Wrachtrup J, Caruso $\mathrm{F}$, et al. Quantum measurement and orientation tracking of fluorescent nanodiamonds inside living cells. Nat Nanotechnol 6(6): 358-363 (2011)

[29] Schirhagl R, Chang K, Loretz M, Degen C L. Nitrogenvacancy centers in diamond: nanoscale sensors for physics and biology. Ann Rev Phys Chem 65(1): 83-105 (2014)

[30] Doherty M W, Manson N B, Delaney P, Jelezko F, Wrachtrup J, Hollenberg LC L. The nitrogen-vacancy colour centre in diamond. Phys Rep 528(1): 1-45 (2013)

[31] Liu Y H, Li G J. A new method for producing "lotus effect" on a biomimetic shark skin. J Colloid Interface Sci $\mathbf{3 8 8}(1)$ : 235-242 (2012)

[32] Lee S-J, Lee S-H. Flow field analysis of a turbulent boundary layer over a riblet surface. Exp Fluids 30(2): 153-166 (2001)

[33] Munson B R, Young D F, Okiishi T H. Fundamentals of fluid mechanics. New York (USA): John Wiley \& Sons, Inc, 1990. 


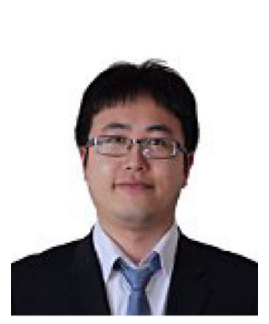

Wei DAI. He received his bachelor and master degrees in materials science and technology from Huazhong University and Science and Technology, in 2009 and 2012

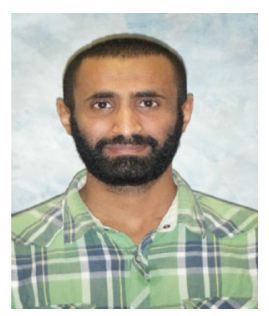

Masfer ALKAHTANI. He received his MSc and PhD from Texas A\&M University in 2014 and 2018, respectively. His research interest includes organic fluorescent nano-

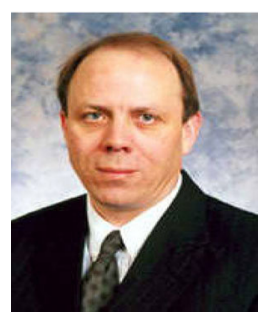

Philip R. HEMMER. He is a professor of the Department of Electrical \& Computer Engineering at Texas A\&M University. Hemmer received his $\mathrm{PhD}$ in physics from MIT in 1984. His research interest includes organic fluorescent nanodiamonds, solid materials for quantum optics, sub-wavelength imaging, single molecule imaging, ultra-sensitive room temperature solid-state magnetometers, nano-fabrication of surface plasmon structures, quantum computing and storage in solid

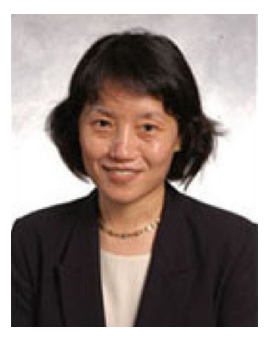

Hong LIANG. She is Oscar S. Wyatt Jr. professor at J. Mike Walker ' 66 Department of Mechanical Engineering, Texas A\&M University. She received her M.S and Ph.D from Stevens Institute of Technology. Her research interests have been in respectively. After that he entered the graduate program at Texas A\&M University and obtained his Ph.D in 2017. His primary research areas have been focused on nanotribology. He is currently with Gränges at Tennessee.

diamonds, high pressure and high temperature diamond growth, nanodiamond surface modifications, solid materials for quantum optics, upconversion nanoparticles, nanofabrication, quantum sensing, magnetometery, and bio-imaging.

materials, quantum communication and teleportation in with solid materials, sensitive chemical/biological agent and IED detection using quantum coherence, ultraslow and stopped light in solids, materials and techniques for resonant nonlinear optics, phaseconjugate-based turbulence aberration compensation, spectral holeburning materials and techniques for ultra-dense memories and high temperature operation, holographic optical memory materials, smart pixels devices, optical correlators, photorefractive applications, atomic clocks, laser trapping and cooling.

fundamental aspects of friction, wear, and lubrication. Her group carries out research in design and synthesis of advanced materials that have unique characteristics to be applied for tribological applications including lubrication, wear protection, and chemical-mechanical polishing. The group develops alternative approaches to probe material surfaces during tribological process. 